\title{
Microtubule-Associated Protein Mdp3 Promotes Breast Cancer Growth and Metastasis
}

\author{
Tala $^{1^{*}}$, Songbo Xie ${ }^{*}$, Xiaodong Sun ${ }^{2 *}$, Xiaoou Sun ${ }^{1}$, Jie Ran ${ }^{1}$, Linlin Zhang1, Dengwen Li ${ }^{1}$, Min Liu1, Gang \\ $\mathrm{BaO}^{2 \bowtie}$, Jun Zhou ${ }^{1,2 \bowtie}$ \\ 1. State Key Laboratory of Medicinal Chemical Biology, College of Life Sciences, Nankai University, Tianjin 300071, China. \\ 2. Department of Biomedical Engineering, Georgia Institute of Technology and Emory University, Atlanta, GA 30332, USA. \\ *These authors contributed equally to this work.
}

\begin{abstract}
$\square$ Corresponding author: Jun Zhou, Ph.D., State Key Laboratory of Medicinal Chemical Biology, College of Life Sciences, Nankai University, Tianjin 300071, China. Telephone: +86-22-2350-4946; Fax: +86-22-2350-4946; E-mail: junzhou@nankai.edu.cn Gang Bao, Ph.D., Department of Biomedical Engineering, Georgia Institute of Technology and Emory University, Atlanta, GA 30332, USA. Telephone: +1-404-385-0373; Fax: +1-404-894-4243; E-mail: gang.bao@bme.gatech.edu.

(c) Ivyspring International Publisher. This is an open-access article distributed under the terms of the Creative Commons License (http://creativecommons.org/ licenses/by-nc-nd/3.0/). Reproduction is permitted for personal, noncommercial use, provided that the article is in whole, unmodified, and properly cited.
\end{abstract}

Received: 2014.05.24; Accepted: 2014.07.29; Published: 20I4.08.15

\begin{abstract}
Breast cancer is the most prevalent cancer in women worldwide with a high mortality rate, and the identification of new biomarkers and targets for this disease is greatly needed. Here we present evidence that microtubule-associated protein (MAP) 7 domain-containing protein 3 (Mdp3) is highly expressed in clinical samples and cell lines of breast cancer. The expression of Mdp3 correlates with clinicopathological parameters indicating breast cancer malignancy. In addition, Mdp3 promotes breast cancer cell proliferation and motility in vitro and stimulates breast cancer growth and metastasis in mice. Mechanistic studies reveal that $\gamma$-tubulin interacts with and recruits Mdp3 to the centrosome and that the centrosomal localization of Mdp3 is required for its activity to promote breast cancer cell proliferation and motility. These findings suggest a critical role for Mdp3 in the growth and metastasis of breast cancer and may have important implications for the management of this disease.
\end{abstract}

Key words: breast cancer, cell proliferation, cell motility, growth, metastasis.

\section{Introduction}

Breast cancer remains the most commonly diagnosed cancer in women, despite substantial progress in its prevention, diagnosis, and treatment ${ }^{1}$. Breast cancer usually originates from the inner lining of mammary ducts and lobules and can metastasize to the bone, lung, liver, and brain ${ }^{2}$. In most cases, the breast-conserving surgery combined with radiation therapy or chemotherapy could effectively manage early breast cancer. However, difficulties still remain in treating patients who have advanced metastatic breast cancer ${ }^{3}$. Although current chemotherapeutic agents largely shrink breast cancer in the beginning, frequent acquisition of drug resistance and severe side effects are major issues of these agents. In addition, some failures in chemotherapy result from low susceptibility caused by the heterogeneous nature of breast cancer. Over the past decades, the identification of biomarkers of breast cancer have shed light on exploring efficient therapeutic strategies against this disease. For example, Herceptin, a monoclonal antibody against human epidermal growth factor receptor 2 (HER2), has benefited patients who are diagnosed with HER2-positive, advanced breast cancer4.

Microtubule-associated proteins (MAPs) represent a family of proteins that regulate microtubule properties and functions ${ }^{5-8}$. Accumulating evidence shows that changes in the expression or post-translational modification of MAPs can contrib- 
ute to the dysregulation of microtubule dynamics and consequently lead to the development of serious diseases, including cancer9-12. The alteration of microtubule dynamics by these changes may also result in tumor resistance to chemotherapeutic agents that target microtubules ${ }^{9}$. Many MAPs with aberrant expression have been depicted to be relevant to susceptibility to microtubule targeting agents ${ }^{13-17}$, and targeting MAPs has been considered as a strategy for rational administration of chemotherapeutic agents against cancer ${ }^{9,18-20}$. MAP7 domain-containing protein 3 (Mdp3) has recently been shown to regulate microtubule assembly and stability via its interaction with tubulin and microtubules ${ }^{21}$. In addition, Mdp3 can control microtubule stability through modulating the activity of histone deacetylase 6 (HDAC6) ${ }^{22}$. However, the physiological and pathological functions of Mdp3 are unclear. In this study, we present the first evidence that Mdp3 plays an important role in breast cancer growth and metastasis.

\section{Materials and Methods}

\section{Materials}

Sulforhodamine B (SRB), 4',6-diamidino-2phenylindole (DAPI) and antibodies against $\beta$-actin, $\gamma$-tubulin, and glutathione S-transferase (GST) were purchased from Sigma-Aldrich. 3-(4, 5-dimethylthiazolyl-2)-2, 5-diphenyltetrazolium bromide (MTT) was from Songon Biotech. Antibody against Mdp3 was generated as described previously ${ }^{21}$. Anti-Ki67 antibody and horseradish peroxidase-conjugated secondary antibodies were from Abcam and Santa Cruz Biotechnology, respectively. Fluorescein- and rhodamine-conjugated secondary antibodies were from Jackson ImmunoResearch Laboratories.

\section{Cell culture}

MCF-10A normal human breast epithelial cells and all human breast cancer cells were purchased from the American Type Culture Collection and cultured according to the manuals. The 4T1-luc luciferase-expressing mouse breast cancer cells were kindly provided by Dr. Zhinan Yin (Nankai University, China) and cultured in RPMI-1640 medium supplemented with $10 \%$ fetal bovine serum.

\section{Plasmids, siRNAs, and transfections}

Mammalian expression plasmids for Mdp3 were generated as described previously ${ }^{21,22}$. Control siRNA (5'-CGUACGCGGAAUACUUCGA-3'), $\quad \gamma$-tubulin siRNA (5'-GGACAUGUUCAAGGACAACUUUGAU $\left.-3^{\prime}\right)$, and human Mdp3 siRNAs (1: 5'-GGAGAGAAGCUCAUCUUUA-3'; 2: 5'-CUUAU UCUCUUGUGUCUAA-3') were synthesized by
Invitrogen. Mouse Mdp3 siRNAs (1: 5'-GCUACCAGUAGCAGCACAU-3'; 2: 5'-GCGACA UAAACGUGGUAUU-3') were synthesized by Ribobio. Plasmids and siRNAs were transfected with the polyethyleneimine reagent (Sigma-Aldrich) and DharmaFect1 reagent (Dharmacon), respectively, according to the manufacturers' manuals.

\section{Human tissue samples}

Samples of human breast cancer and adjacent tissues were obtained from patients who underwent surgical resection at Shanxian Dongda Hospital, Shandong, China. The use of human tissues was approved by our Institutional Ethics Committee. Tissues were immunostained as described previously ${ }^{11}$. Briefly, sections were deparaffinized and rehydrated with xylene and graded alcohols. After antigen retrieval and inactivation of endogenous peroxidase, sections were blocked with goat serum and incubated with primary antibodies. Subsequently the sections were incubated with biotinylated secondary antibody and streptavidin-biotin-peroxidase, using daminobenzidine as the chromogen substrate, and finally haematoxylin counterstaining were performed. The expression of Mdp3 was graded according to the staining intensity $(0=$ negative; $1=$ low; $2=$ medium; $3=$ high $)$ and the percentage of stained cells $(0=0 \%$ stained; $1=1 \%-25 \%$ stained; $2=26 \%-50 \%$ stained $; 3=$ $51 \%-100 \%$ stained) as described previously ${ }^{16}$.

\section{Immunoblot analysis}

Proteins were separated by sodium dodecyl sulfate-polyacrylamide gel electrophoresis and transferred onto polyvinylidene difluoride membranes (Millipore), followed by blocking with Tris-buffered saline containing $0.2 \%$ Tween 20 and $5 \%$ fat-free dry milk. Membranes were incubated sequentially with primary antibodies and horseradish peroxidase-conjugated secondary antibodies. Specific proteins were detected with the enhanced chemiluminescence detection reagent (Pierce), according to the manufacturer's protocol.

\section{GST pull-down and immunoprecipitation}

For GST pull-down, cells transfected with GST-tagged plasmids were lyzed and cell lysates were incubated with glutathione-conjugated agarose beads at $4{ }^{\circ} \mathrm{C}$ overnight. The pulled-down proteins were then analyzed by immunoblotting. For immunoprecipitation, the cell lysates were incubated with anti- $\gamma$-tubulin antibody or control IgG at $4{ }^{\circ} \mathrm{C}$ for 2 hours, and protein A/G agarose beads were then added to the solution for another 2 hours. The precipitated proteins were then examined by immunoblotting. 


\section{Cell proliferation and colony formation}

Cells were seeded in 96-well plates, and the density of cells was determined by SRB and MTT assays. For cell cycle analysis, cells were collected and washed twice with ice-cold phosphate-buffered saline (PBS). Then cells were fixed with $70 \%$ ethanol at $4{ }^{\circ} \mathrm{C}$ for 24 hours and incubated with propidium iodide (PI)/RNase A solution for 30 minutes. Samples were analyzed with the BD FACS Calibur flow cytometer. For colony formation assays, cells were seeded in six-well plates and incubated for 2 weeks. The colonies were then fixed with methanol and stained with $0.1 \%$ crystal violet. Photographs were taken and the number of colonies at each well was counted.

\section{Cell motility assays}

Cell motility assays were performed as described previously ${ }^{8}$. For wound healing experiments, cells cultured in 24-well plates were scratched with a $10 \mu \mathrm{L}$ pipette tip to generate the wound. Cells were then washed twice with PBS to remove the cell debris. Photographs of the wound were taken at 0 hour and 24 hours to determine the extent of wound closure. For transwell experiments, cells suspended in serum-free medium were added to the upper chamber of inserts that were precoated with matrigel, fibronectin, poly-D-lysine, or gelatin. Cells on the inside of the inserts were removed 24 hours later with a cotton swab, and invaded cells underside were fixed with $4 \%$ paraformaldehyde, stained with the crystal violet solution, and imaged. For cell tracking assays, cells were cultured in a 35-mm dish overnight. The migration of the cells was recorded at an interval of 5 minutes for 12 hours with a phase contrast microscope. Tracks and migration velocities were determined with the Image-J software.

\section{Tumor growth and metastasis in mice}

All procedures for mouse care and operation were carried out according to the guidelines of experimental animals. The use of mice was approved by our Institutional Animal Care Committee. To examine tumor growth, cells were injected subcutaneously into the right flank of female athymic nude mice, and tumor volumes were measured. After the mice were sacrificed, tumors were isolated, photographed, weighed and sectioned for Ki67 immunostaining. To analyze tumor metastasis, 4T1-luc cells were injected into the lateral tail vein of female BALB/c mice. Luciferin $(150 \mathrm{mg} / \mathrm{kg})$ was injected intraperitoneally 10 minutes before imaging. Metastatic cells were monitored with an IVIS Imaging System (Xenogen). At the endpoint of experiments, mice were sacrificed and tumors were harvested for bioluminescent imaging or sectioned for haematoxylin and eosin staining.

\section{Immunofluorescence microscopy}

Cells grown on glass coverslips were fixed with methanol at $-20{ }^{\circ} \mathrm{C}$ for 5 minutes and then blocked with $2 \%$ bovine serine albumin in PBS for 20 minutes. Cells were incubated with primary antibodies and then with fluorescein- and rhodamine-conjugated secondary antibodies. Nuclei were stained with DAPI. Coverslips were mounted with $90 \%$ glycerol in PBS and then examined with an Axio Observer A1 fluorescence microscope (Carl Zeiss, Inc.).

\section{Results}

\section{Mdp3 is highly expressed in human breast cancer tissues and cell lines}

We obtained 85 breast cancer tissue samples and 49 adjacent tissue samples from patients who underwent surgical resection and examined Mdp3 expression by immunohistochemical staining. The samples were classified into four groups according to the staining intensity and the percentage of stained cells (Figures 1A and 1B). We found that $43.5 \%$ of breast cancer tissues showed high expression (+++) of Mdp3, whereas only $20.4 \%$ of the adjacent issues had high expression (Figure 1C).

To study whether Mdp3 expression is linked to the clinical characteristics of breast cancer, we analyzed several clinicopathological parameters of these 85 breast cancer tissue samples. We found that Mdp3 expression correlated with higher pathological tumor node metastasis (pTNM) stage (Figure 1D), higher histological grade (Figure 1E), and higher incidence of lymph node metastasis (Figure 1F). In contrast, a negative correlation was observed between Mdp3 and estrogen receptor (Figure 1G), and between Mdp3 and progesterone receptor (Figure $1 \mathrm{H}$ ). No significant correlation was identified between Mdp3 and the age of patients.

We then performed immunoblotting to examine the expression of Mdp3 in MCF-10A normal breast epithelial cells and several breast cancer cell lines. As shown in Figure 1I, Mdp3 was hardly detectable in normal breast epithelial cells, whereas its expression was significantly increased in all the breast cancer cell lines examined except SW527 cells. These data indicate a potential role for elevated expression of Mdp3 in the pathogenesis of breast cancer.

\section{Mdp3 promotes the proliferation of breast cancer cells}

To investigate how Mdp3 is involved in breast cancer, we examined its role in breast cancer cell proliferation by knocking down its expression with specific siRNAs (Figure 2A). By SRB and MTT assays, we found that depletion of Mdp3 significantly sup- 
pressed the proliferation of breast cancer cells (Figures 2B and 2C). Mdp3 siRNAs also remarkably decreased the number of colonies formed from breast cancer cells, as compared to the control group (Figure 2D). To further analyze the role of Mdp3 in breast cancer cell proliferation, we overexpressed Mdp3 in MCF-10A normal breast epithelial cells and BT549 breast cancer cells (Figure 2E). We found that overexpression of Mdp3 could promote the formation of colonies from MCF-10A and BT549 cells (Figure 2F). By flow cytometric analysis of DNA content, we found that knockdown of Mdp3 expression did not significantly affect the percentages of breast cancer cells at G1, S, and G2/M phases (Figures 2G and 2H).
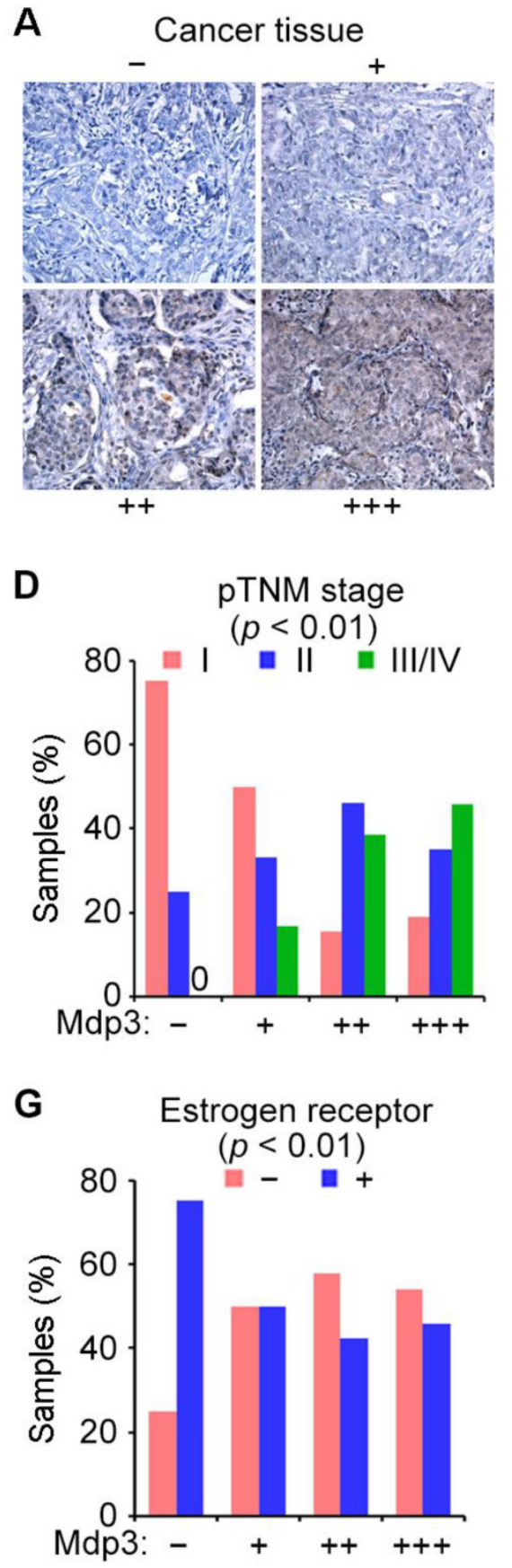
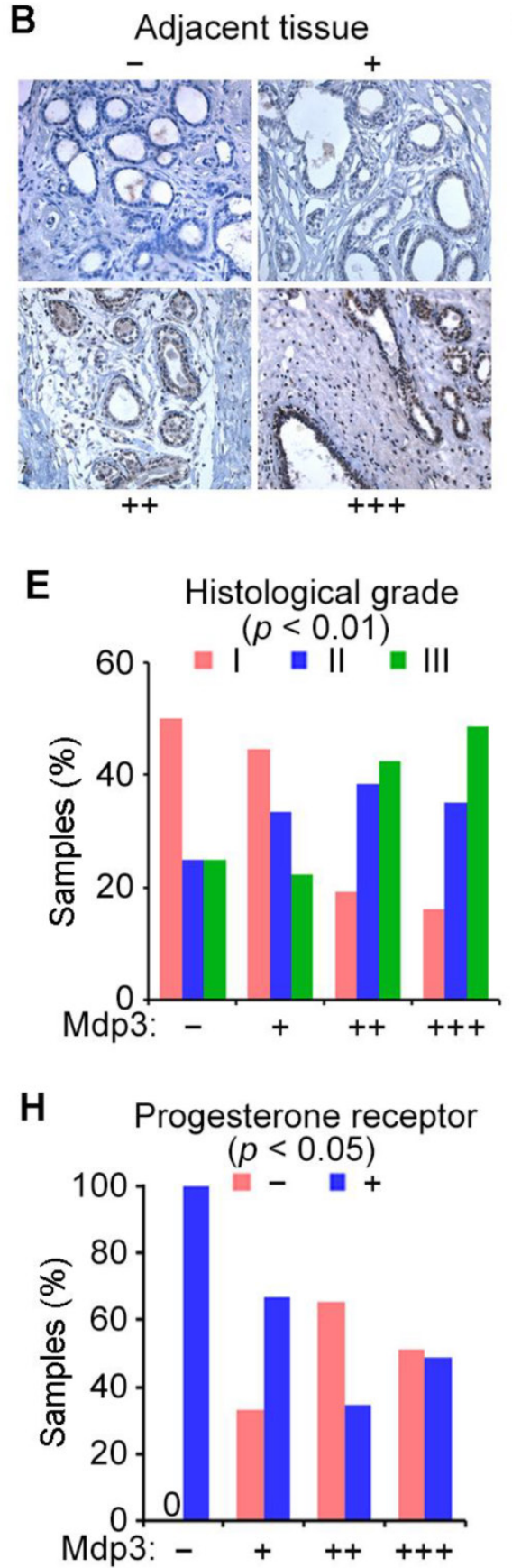
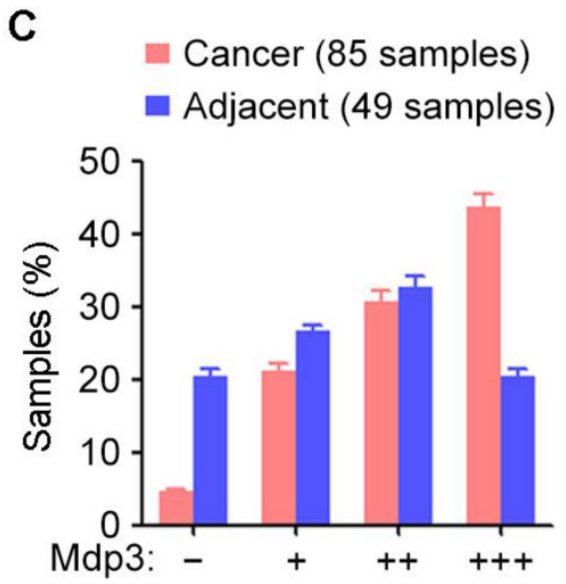

$\mathbf{F}$

Lymph node metastasis

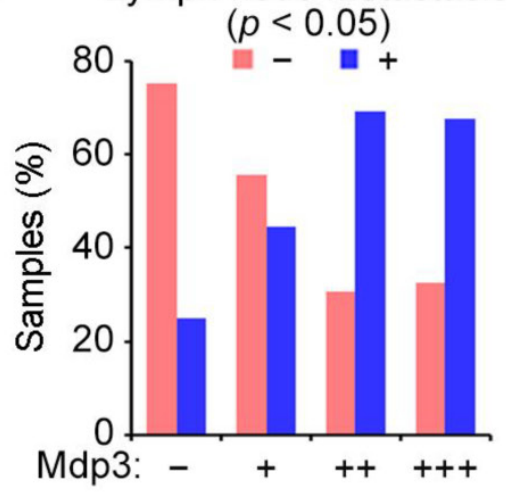

Figure I. Mdp 3 is highly expressed in human breast cancer tissues and cell lines. (A and B) Immunohistochemical staining of Mdp3 in breast cancer tissues ( $A$ ) and adjacent tissues (B), with negative (-), low (+), medium (++), and high (+++) expression. (C) Quantitative analysis of Mdp3 expression in 85 breast cancer tissue samples and 49 adjacent tissue samples. (D-H) Analysis of the correlations between Mdp3 expression and clinicopathological parameters indicating the malignancy of breast cancer, including the pathological tumor node metastasis (PTNM) stage (D), histological grade (E), lymph node metastasis (F), estrogen receptor (G), and progesterone receptor (H). (I) Immunoblot analysis of Mdp3 and $\beta$-actin expression in MCF-IOA normal breast epithelial cells and breast cancer cell lines. 

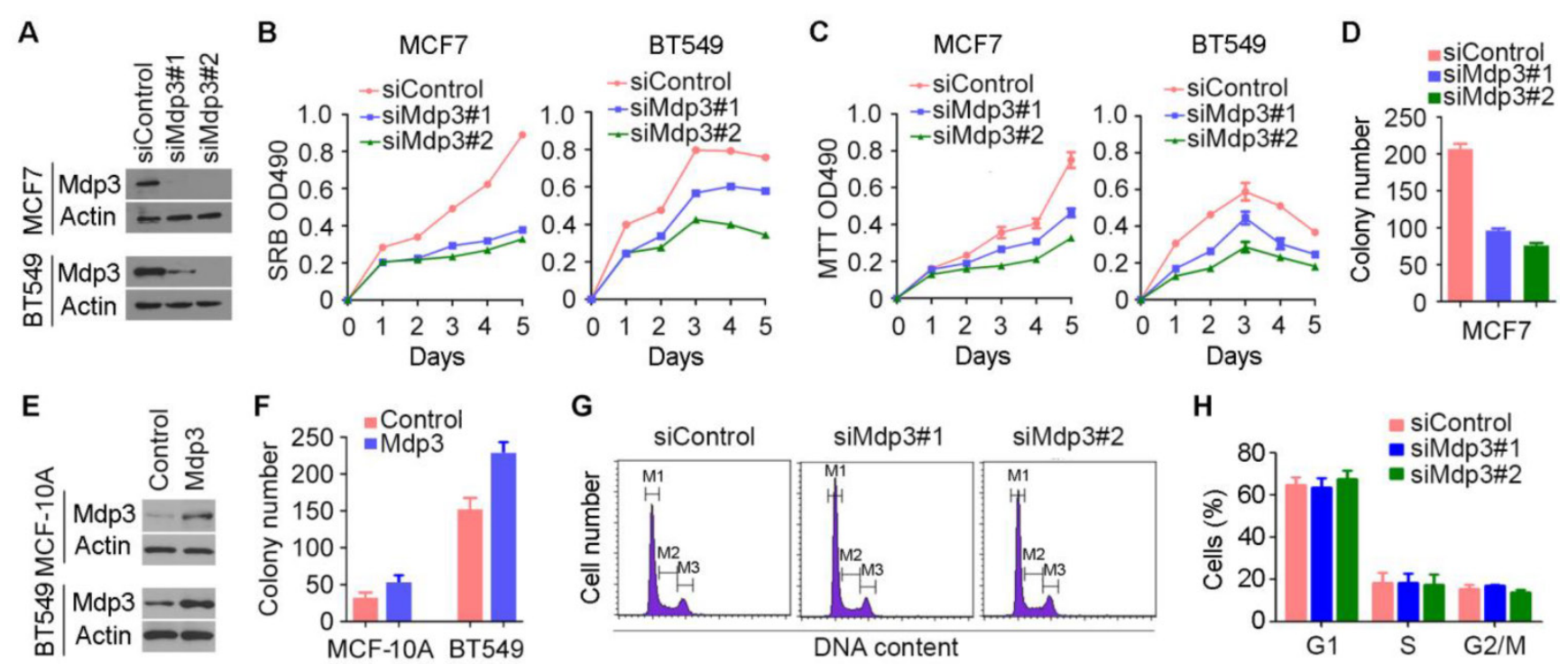

Figure 2. Mdp3 promotes the proliferation of breast cancer cells. (A) Immunoblot analysis of Mdp3 and $\beta$-actin expression in MCF7 and BT549 cells transfected with control or Mdp3 siRNAs for 48 hours. (B and C) Determination of cell proliferation by SRB (B) and MTT (C) assays in cells transfected with control or Mdp3 siRNAs. (D) Quantification of colonies formed from control or Mdp3 siRNA-transfected MCF7 cells. (E) Immunoblot analysis of Mdp3 and $\beta$-actin expression in MCF-I0A and BT549 cells transfected with control or Mdp3 for 48 hours. (F) Quantification of colonies formed from control or Mdp3-transfected cells. (G) MCF7 cells were transfected with control or Mdp3 siRNAs, and the DNA content was analyzed by flow cytometry. The MI, M2, and M3 gates indicate cells in $G_{1}, S$, and G2/M phases, respectively. $(H)$ Quantification of the percentages of cells in $G_{1}, S$, and $G 2 / M$ phases.

\section{Mdp3 stimulates the motility of breast cancer cells}

We then performed wound healing and transwell assays to study the potential involvement of Mdp3 in breast cancer cell motility. We found that siRNA-mediated depletion of Mdp3 expression dramatically inhibited the extent of wound closure in both MDA-MB-231 and Hs578T cells (Figures 3A-C). In addition, Mdp3 siRNAs significantly compromised the invasion of breast cancer cells through matrigel-coated membranes (Figures $3 \mathrm{D}$ and $3 \mathrm{E}$ ). The requirement of Mdp3 for cell invasion was also observed when the membrane was coated with fibronectin, poly-D-lysine, or gelatin (Figure $3 \mathrm{~F}$ ). To gain more insight into the role of Mdp3 in breast cancer cell motility, we tracked breast cancer cells and analyzed their migration velocity. Cell movement was recorded by time-lapse imaging, and the movement velocity was determined by regression analysis of the accumulated distance versus time. We found that knockdown of Mdp3 expression decreased the accumulated distance of cell movement and reduced the velocity (Figures 3G-I). By wound healing and transwell experiments, we further found that overexpression of Mdp3 could enhance the motility of MCF-10A and MDA-MB-231 cells (Figures 3J-L). Collectively, these data demonstrate a critical role for Mdp3 in breast cancer cell motility.

\section{Mdp3 increases breast cancer growth and metastasis in mice}

To investigate the role of Mdp3 in vivo, control or
Mdp3 siRNA-transfected MDA-MB-231 cells were injected subcutaneously into athymic nude mice, and tumor volume was then measured each week. We found that the volume of tumors in Mdp3 siRNA groups was markedly smaller than that in the control group (Figure 4A). In addition, the weight of tumors isolated from the Mdp3 siRNAs groups was decreased as compared to the control group (Figure 4B). We also found that overexpression of Mdp3 increased tumor volume and weight in mice (Figures $4 \mathrm{C}$ and $4 \mathrm{D})$. Immunohistochemical staining further revealed that the expression of the cell proliferation marker Ki67 in tumors was significantly lower in the Mdp3 siRNA group than that in the control group (Figures $4 \mathrm{E}$ and $4 \mathrm{~F})$.

We then used mouse 4T1-luc cells to evaluate the role of Mdp3 in breast cancer metastasis in mice. We injected control or mouse Mdp3 siRNA-transfected 4T1-luc cells into the tail vein, and luciferin was injected to allow the detection of 4T1-luc cells via bioluminescence imaging. We found that knockdown of Mdp3 expression greatly decreased the metastasis of 4T1-luc cells to the lung (Figures 4G-I). Measurement of the luminescence photon flux in the isolated tumors also showed that Mdp3 was important for cancer metastasis (Figure 4J). We then examined the lung sections by hematoxylin and eosin staining. As shown in Figures $4 \mathrm{~K}$ and $4 \mathrm{~L}$, Mdp3 siRNAs remarkably reduced the percentage of the metastasis area in the lung. In addition, overexpression of Mdp3 increased the percentage of the metastasis area in the lung (Figure 4M). Thus, Mdp3 enhances breast cancer 
growth and metastasis in mice.

\section{Interaction of Mdp3 with Y-tubulin is required for its ability to promote breast cancer cell proliferation and motility}

To provide mechanistic insight into the role of Mdp3 in breast cancer, we examined its subcellular localization in breast cancer cells by immunofluorescence microscopy. Consistent with the previous finding 21 , Mdp3 was partially colocalized with microtubules (Figure 5A). Interestingly, Mdp3 signal was also detected at the centrosome (Figures 5A and 5B). By
GST pull-down assay, we found that Mdp3 full-length, but not its C-terminus, interacted with the centrosomal protein $\gamma$-tubulin (Figure 5C). By immunoprecipitation experiments, we further found an interaction between endogenous Mdp3 and $\gamma$-tubulin in breast cancer cells (Figure 5D). Immunofluorescence microscopy showed that GFP-Mdp3 full-length was partially colocalized with $\gamma$-tubulin and exhibited microtubule-like filaments, whereas its C-terminus had a dispersed distribution pattern in the cell (Figure $5 \mathrm{E})$.
A

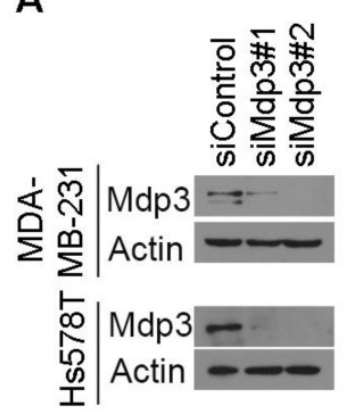

C

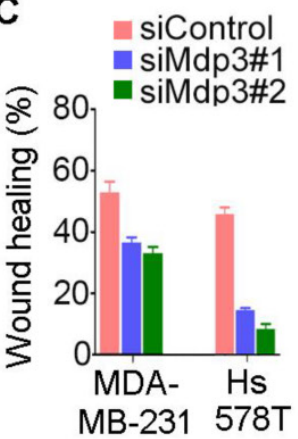

$\mathbf{J}$

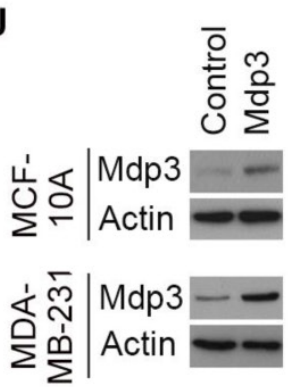

B
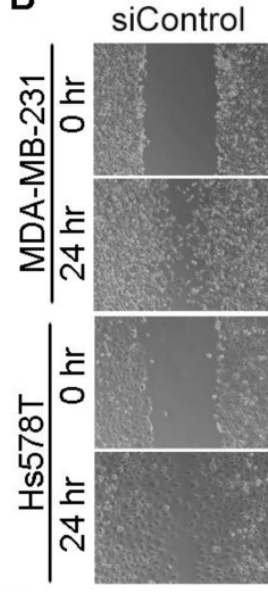

D

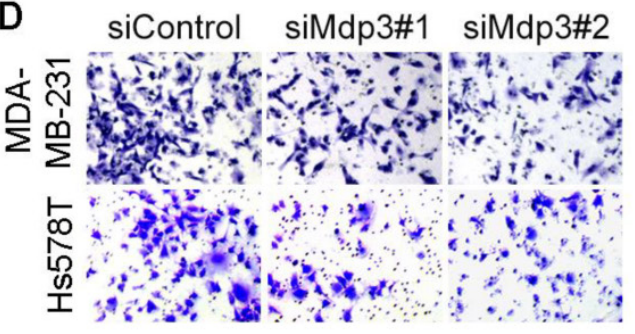

K

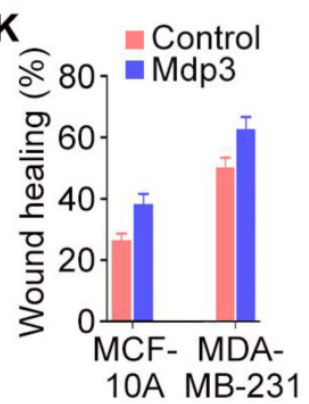

L Control

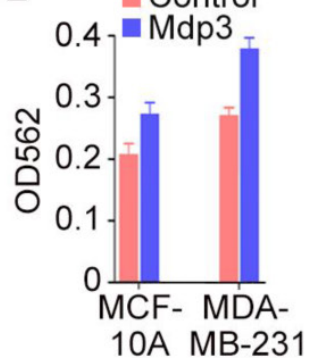

E

$\mathbf{F}$

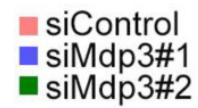

siControl

siMdp3\#1

- siMdp3\#2
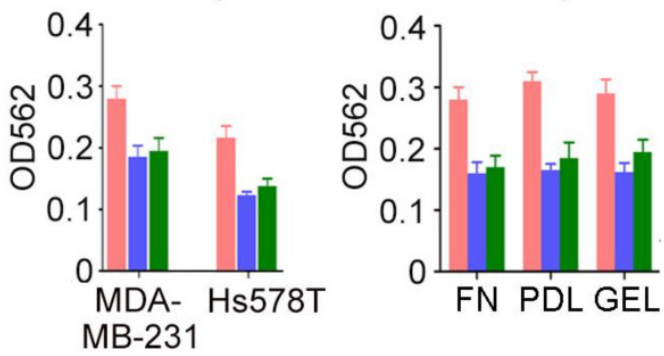

G

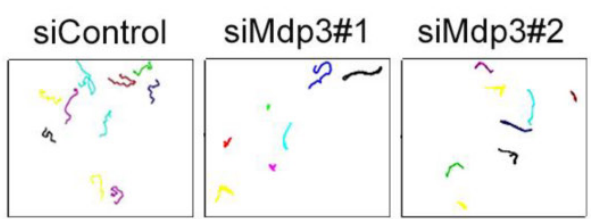

H

Euclidean distance Accumulated distance

siControl

- siMdp3\#1

- siMdp3\#2
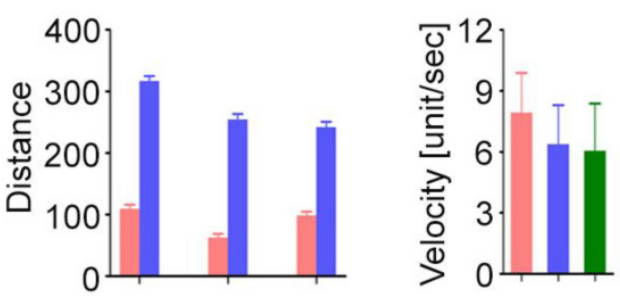

Figure 3. Mdp3 stimulates the motility of breast cancer cells. (A) Immunoblot analysis of Mdp3 and $\beta$-actin expression in MDA-MB-23I and Hs578T cells transfected with control or Mdp3 siRNAs for 48 hours. (B) Control or Mdp3 siRNA-transfected cells were scratched, and wound margins were imaged 0 or 24 hours later. (C) Experiments were performed as in B, and the extent of wound closure was analyzed. (D) Control or Mdp3 siRNA-transfected cells were plated into inserts precoated with matrigel, and cells underside the porous membrane were stained with crystal violet 24 hours later. (E) Experiments were performed as in $D$, and the extent of cell invasion was quantified. (F) Experiments were performed as in D, except that the inserts were precoated with fibronectin (FN), poly-D-lysine (PDL), or gelatin (GEL). (G) Movement tracks of MDA-MB-23। cells transfected with control or Mdp3 siRNAs. $(\mathrm{H})$ Experiments were performed as in $\mathrm{G}$, and the Euclidean distance and the accumulated distance were determined. (I) Experiments were performed as in panel G, and the velocity of cell movement was calculated. (J) Immunoblot analysis of Mdp3 and $\beta$-actin expression in MCF-I0A and MDA-MB-23I cells transfected with control or Mdp3 for 48 hours. (K) Control or Mdp3-transfected cells were scratched, and the extent of wound closure was analyzed 24 hours later. (L) Control or Mdp3-transfected cells were plated into inserts precoated with matrigel, and the extent of cell invasion was quantified by crystal violet staining 24 hours later. 
A

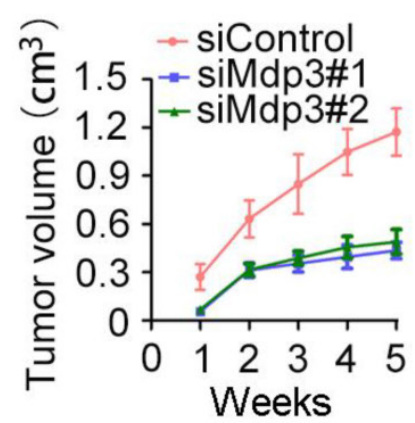

E

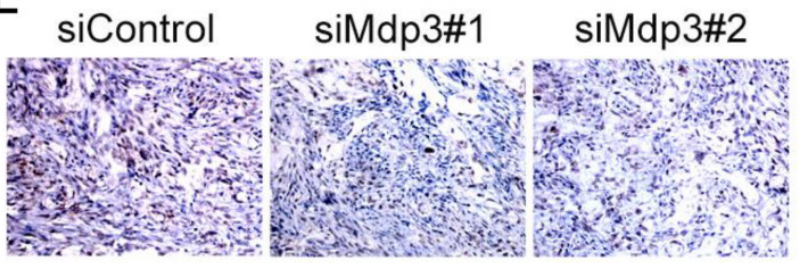

$\mathrm{H}$

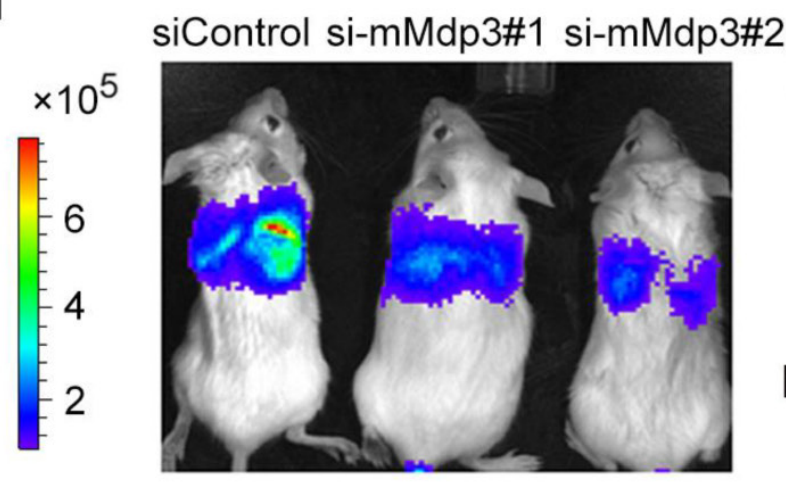

$\mathbf{J}$

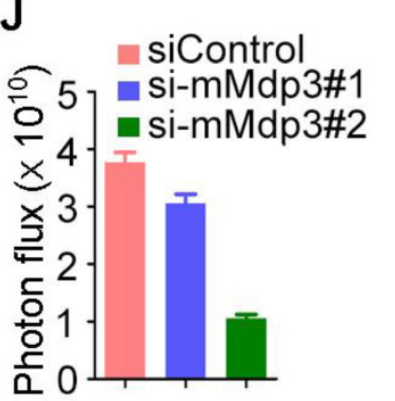

B

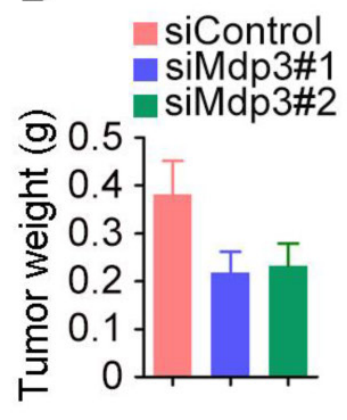

siMdp3\#2
F siControl

C

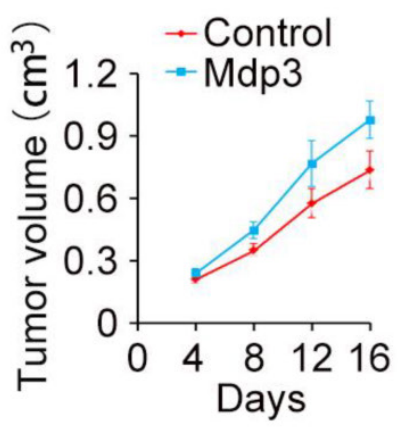
- siMdp3\#1 siMdp3\#2

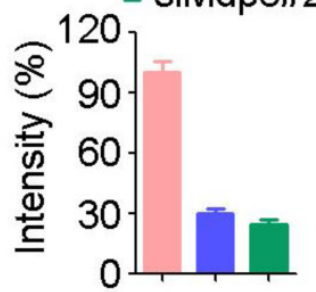

D

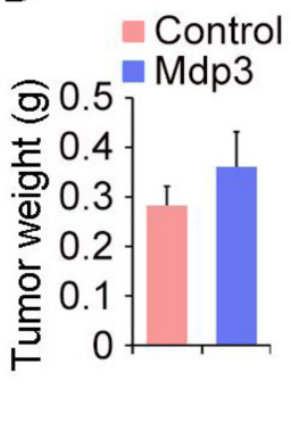

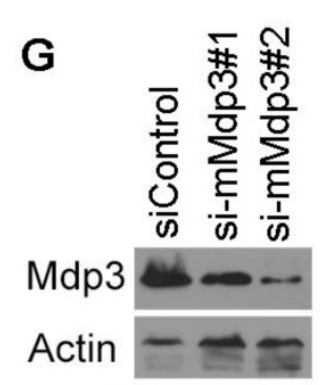

4T1-luc cells

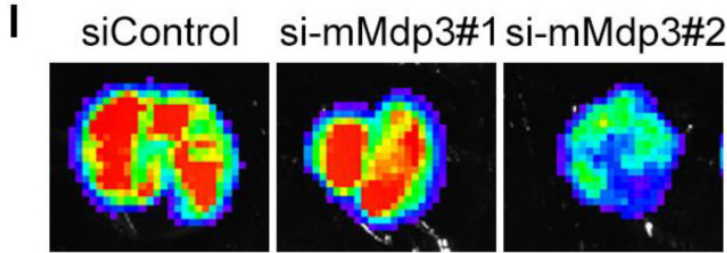

K

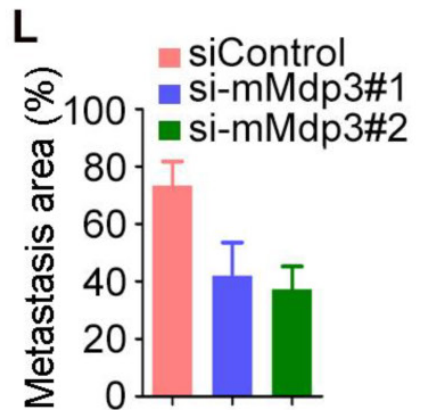

Figure 4. Mdp3 increases breast cancer growth and metastasis in mice. (A) MDA-MB-23I cells were transfected with control or Mdp3 siRNAs, and the transiently-silenced cells were injected subcutaneously into the right flanks of female athymic nude mice (7 mice per group). Tumor volume was measured each week. (B) Experiments were performed as in A, and mice were sacrificed 5 weeks post-injection. Tumors were then isolated and weighed. (C) MDA-MB-23I cells were transfected with control or Mdp3, and the transiently-overexpressed cells were injected subcutaneously into the right flanks of female athymic nude mice (6 mice per group). Tumor volume was measured every 4 days. (D) Experiments were performed as in C, and mice were sacrificed 16 days post-injection. Tumors were then isolated and weighed. (E) Immunohistochemical staining of Ki67 in tumors. (F) Experiments were performed as in E, and the intensity of Ki67 was measured. (G) Immunoblot analysis of Mdp3 and $\beta$-actin expression in 4TI-luc cells transfected with control or mouse Mdp3 siRNAs. (H) 4TI-luc cells transiently silenced with control or mouse Mdp3 siRNAs were injected into BALB/c mice (4 mice per group), and the mice were examined by a bioluminescence imaging instrument 8 days later. (I) Experiments were performed as in $\mathrm{H}$, and mice were sacrificed at the endpoint of day 8 . Tumors were isolated and analyzed with bioluminescence imaging. (J) Experiments were performed as in I, and photon flux was determined. (K) Hematoxylin and eosin staining of lung sections in mice injected with control or mouse Mdp3 siRNA-transfected 4TI-luc cells. (L) Experiments were performed as in $\mathrm{K}$, and the percentage of metastasis area was quantified. (M) $4 \mathrm{TI}$-luc cells transiently transfected with control or Mdp3 were injected into BALB/c mice (6 mice per group). Lung sections were examined by hematoxylin and eosin staining as in $\mathrm{K}$, and the percentage of metastasis area was quantified. 
A

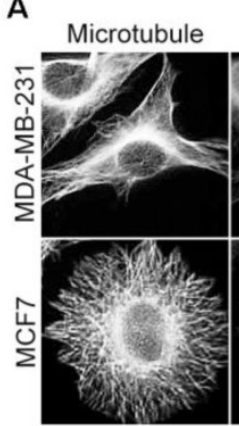

C

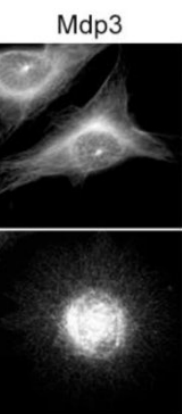

D

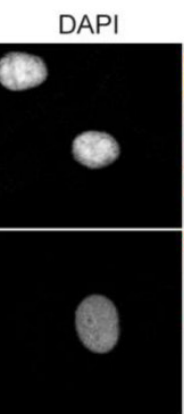

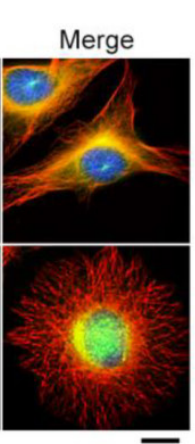

B
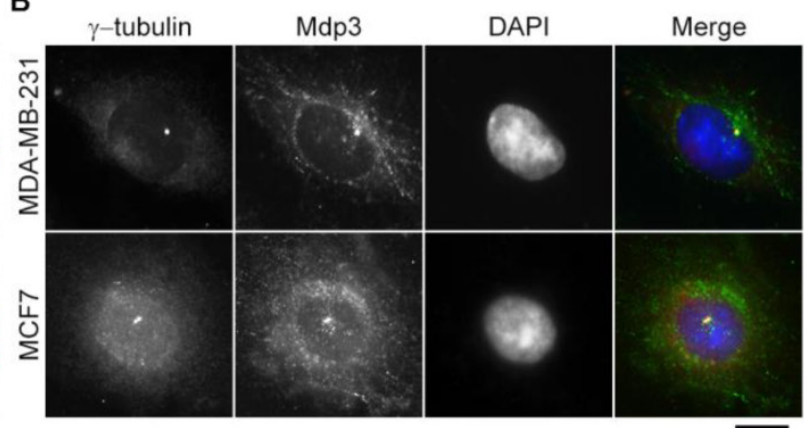

E

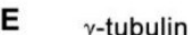

GFP
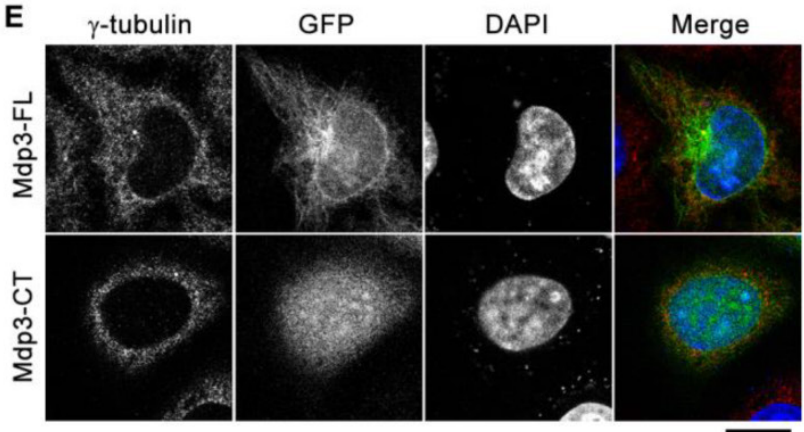

G

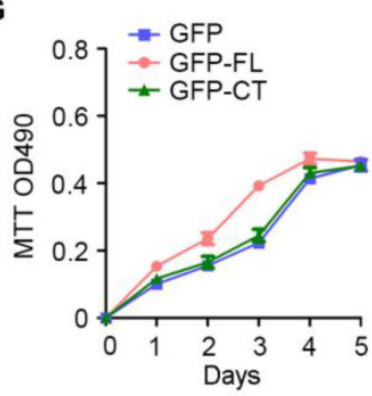

H

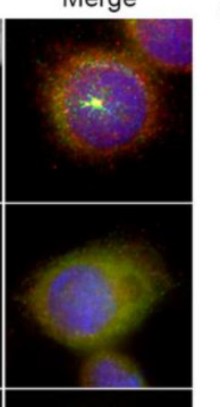

I

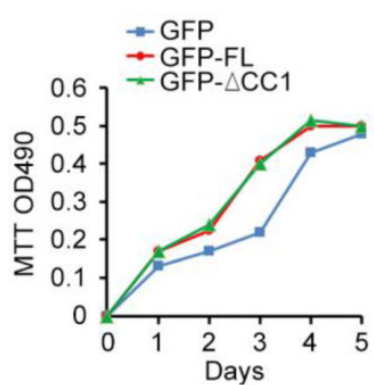

GFP

$=$ GFP-FL

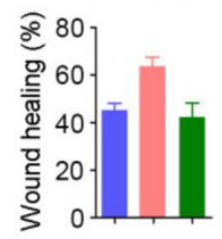

J
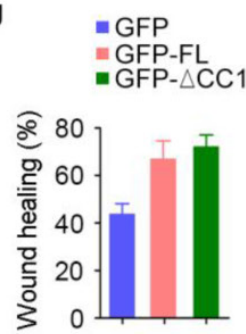

Figure 5. Interaction of Mdp3 with $\mathrm{Y}$-tubulin is required for its ability to promote breast cancer cell proliferation and motility. (A) Immunofluorescence microscopy of microtubules (red), Mdp3 (green), and nuclei (blue) in MDA-MB-23I and MCF7 cells. Scale bar, $10 \mu \mathrm{m}$. (B) Immunofluorescence microscopy of Y-tubulin (red), Mdp3 (green), and nuclei (blue) in MDA-MB-23I and MCF7 cells. Scale bar, $10 \mu \mathrm{m}$. (C) Cells were transfected with GST, GST-Mdp3 full-length (FL), or GST-Mdp3 C-terminus (CT). GST pull-down and immunoblotting were then performed with anti- $\gamma$-tubulin and anti-GST antibodies. (D) Examination of the interaction between endogenous Mdp3 and $Y$-tubulin by immunoprecipitation and immunoblotting. (E) Immunofluorescence staining of Y-tubulin (red) and nuclei (blue) in cells transfected with GFP-Mdp3-FL or GFP-Mdp3-CT (green). Scale bar, $10 \mu \mathrm{m}$. (F) Immunofluorescence staining of $\mathrm{Y}$-tubulin (red), Mdp3 (green), and nuclei (blue) in BT549 cells transfected with control, Y-tubulin, or Mdp3 siRNAs. Scale bar, $10 \mu \mathrm{m}$. (G and H) BT549 cells were transfected with Mdp3 siRNA and GFP, GFP-Mdp3-FL, or GFP-Mdp3-CT. Cell proliferation was then determined with MTT assays (G), and cell migration was determined with wound healing assays $(\mathrm{H})$. (I and J) BT549 cells were transfected with GFP, GFP-Mdp3-FL, or GFP-Mdp3- $\Delta$ CCI (lacking the first coiled coil domain). Cell proliferation was then determined with MTT assays (I), and cell migration was determined with wound healing assays (J).

Interestingly, depletion of $\gamma$-tubulin abolished the centrosomal localization of Mdp3, whereas depletion of Mdp3 did not affect the localization of $\gamma$-tubulin (Figure 5F). Furthermore, Mdp3 full-length, but not its C-terminus, partially restored Mdp3 siRNA-induced proliferation and motility defects of breast cancer cells (Figures 5G and 5H). We also found that deletion of the first coiled coil domain of Mdp3, which mediates its interaction with HDAC6 ${ }^{22}$, did not significantly affect its ability to promote breast cancer cell proliferation and motility (Figures 5I and 5J). Taken together, these data indicate that Mdp3 is recruited to the centrosome through its binding to $\gamma$-tubulin, and that the interaction between Mdp3 and 
$\mathrm{Y}$-tubulin is necessary for Mdp3 to promote breast cancer cell proliferation and motility.

\section{Discussion}

Breast cancer is a multifaceted disease with a variety of biological subtypes and can metastasize to organs such as the bone and lung through the vascular and lymphatic systems. Proteins with abnormal expression or activity in breast cancer cells are thought to have diagnostic and therapeutic potential. The discovery of estrogen receptor, progesterone receptor, and HER2 in the development of breast cancer has benefited many patients. In addition, selective targeting of breast cancer cells by some small molecules may hold promise in a subset of patients ${ }^{23}$. However, more biomarkers and targets for the management of this disease are still needed. This work reveals that Mdp3 is highly expressed in human breast cancer tissues and cell lines and that Mdp3 promotes breast cancer growth and metastasis by stimulating cancer cell proliferation and motility. The potential of Mdp3 as a biomarker or target for the management of breast cancer thus merits further investigation.

Cell motility is known to require the dynamic properties of microtubules ${ }^{24}$, and MAPs are critical for cell motility due to their ability to regulate microtubule dynamics ${ }^{5}$. For example, end-binding protein 1 (EB1), the microtubule-associated deubiquitinase cylindromatosis (CYLD), and the tubulin deacetylase HDAC6 play important roles in the movement of diverse types of cells7,8,25-27. Interestingly, Mdp3 regulates microtubule dynamics through modulating the activity of HDAC6 as a tubulin deacetylase, in addition to its direct binding to microtubules ${ }^{21,22}$. In this study, we demonstrate the localization of Mdp3 to the centrosome in breast cancer cells via its interaction with $\gamma$-tubulin. Given that the centrosome is involved in organelle positioning, cell polarity, cell motility, and mitotic spindle assembly $28-30$, it is possible that Mdp3 regulates breast cancer growth and metastasis through its centrosomal localization and functions.

At present, the precise mechanism of how the Mdp3/ $\gamma$-tubulin axis is connected to the function of Mdp3 in breast cancer cell proliferation and motility is unknown. Our data show that overexpression or knockdown of Mdp3 does not obviously affect $\gamma$-tubulin localization. It is possible that Mdp3 might modulate the interaction of $\gamma$-tubulin with other centrosomal proteins mediating cell proliferation and migration. Interestingly, although Mdp3 promotes microtubule stability partially through its suppression of HDAC6 activity ${ }^{22}$, deletion of the HDAC6-interacting domain of Mdp3 does not significantly influence its effects on breast cancer cell pro- liferation and migration. This finding suggests that HDAC6 might play a minor role, if there is any, in mediating the functions of Mdp3 in breast cancer.

It is noteworthy that breast cancer 1 (BRCA1), a protein commonly mutated in breast and ovarian cancers $^{31}$, is also localized to the centrosome and suppresses microtubule nucleation through ubiquitination of $\gamma$-tubulin ${ }^{32}$. BRCA1 has also been reported to play a critical role in regulating cancer sensitivity to microtubule-targeting agents ${ }^{33}$. In addition, a number of MAPs have been shown to control the sensitivity of paclitaxel in breast cancer via microtubule-dependent mechanisms. For example, overexpression of tau in breast cancer patients leads to poor response to paclitaxel ${ }^{14}$, and the elevation of cytoplasmic linker protein of $170 \mathrm{kDa}$ (CLIP-170) expression enhances breast cancer sensitivity to paclitaxe ${ }^{15}$. Given the increased expression of Mdp3 in breast cancer and its interaction with microtubules and $\gamma$-tubulin, it would be interesting to investigate in the future whether Mdp3 could modulate the sensitivity of breast cancer cells to microtubule-targeting agents.

\section{Abbreviations}

MAP: microtubule-associated protein; Mdp3: MAP7 domain-containing protein 3; HER2: human epidermal growth factor receptor 2; HDAC6: histone deacetylase 6; SRB: sulforhodamine B; DAPI: 4',6-diamidino-2-phenylindole; GST: glutathione S-transferase; MTT: 3-(4, 5-dimethylthiazolyl-2)-2, 5-diphenyltetrazolium bromide; PBS: phosphate-buffered saline; pTNM: pathological tumor node metastasis; CYLD: cylindromatosis; EB1: end-binding protein 1; BRCA1: breast cancer 1; CLIP-170: cytoplasmic linker protein of $170 \mathrm{kDa}$.

\section{Acknowledgements}

This work was supported by the National Basic Research Program of China (2012CB945002 to JZ), the National Natural Science Foundation of China (31130015 to JZ, 31271437 to ML, and 31371382 to DL), the 111 project of the Ministry of Education of China (B08011 to JZ), and the US National Science Foundation as Science and Technology Center Grant (CBET-0939511 to GB).

\section{Competing Interests}

The authors have declared that no competing interest exists.

\section{References}

1. Jemal A, Bray F, Center MM, et al. Global cancer statistics. CA Cancer J Clin. 2011; 61: 69-90.

2. Li CI, Uribe DJ, Daling JR. Clinical characteristics of different histologic types of breast cancer. Br J Cancer. 2005; 93: 1046-52.

3. Lu J, Steeg PS, Price JE, et al. Breast cancer metastasis: challenges and opportunities. Cancer Res. 2009; 69: 4951-3. 
4. Stern HM. Improving treatment of HER2-positive cancers: opportunities and challenges. Sci Transl Med. 2012; 4: 127rv2.

5. Amos LA, Schlieper D. Microtubules and maps. Adv Protein Chem. 2005; 71: 257-98.

6. Yang Y, Liu M, Li D, et al. CYLD regulates spindle orientation by stabilizing astral microtubules and promoting dishevelled-NuMA-dynein/dynactin complex formation. Proc Natl Acad Sci U S A. 2014; 111: 2158-63.

7. Li D, Gao J, Yang Y, et al. CYLD coordinates with EB1 to regulate microtubule dynamics and cell migration. Cell Cycle. 2014; 13: 974-83.

8. Gao J, Huo L, Sun X, et al. The tumor suppressor CYLD regulates microtubule dynamics and plays a role in cell migration. J Biol Chem. 2008; 283: 8802-9.

9. Bhat KM, Setaluri V. Microtubule-associated proteins as targets in cancer chemotherapy. Clin Cancer Res. 2007; 13: 2849-54.

10. Liu M, Wang $X$, Yang $Y$, et al. Ectopic expression of the microtubule-dependent motor protein Eg5 promotes pancreatic tumourigenesis. J Pathol. 2010; 221: 221-8.

11. Dong $X$, Liu F, Sun L, et al. Oncogenic function of microtubule end-binding protein 1 in breast cancer. J Pathol. 2010; 220: 361-9.

12. Sun L, Gao J, Dong X, et al. EB1 promotes Aurora-B kinase activity through blocking its inactivation by protein phosphatase 2A. Proc Natl Acad Sci U S A. 2008; 105: 7153-8.

13. Alli E, Bash-Babula J, Yang JM, et al. Effect of stathmin on the sensitivity to antimicrotubule drugs in human breast cancer. Cancer Res. 2002; 62: 6864-9.

14. Rouzier R, Rajan R, Wagner P, et al. Microtubule-associated protein tau: a marker of paclitaxel sensitivity in breast cancer. Proc Natl Acad Sci U S A. 2005; 102: 8315-20.

15. Sun X, Li D, Yang Y, et al. Microtubule-binding protein CLIP-170 is a mediator of paclitaxel sensitivity. J Pathol. 2012; 226: 666-73.

16. Wang H, Liu B, Zhang C, et al. Parkin regulates paclitaxel sensitivity in breast cancer via a microtubule-dependent mechanism. J Pathol. 2009; 218: 76-85.

17. Luo Y, Li D, Ran J, et al. End-binding protein 1 stimulates paclitaxel sensitivity in breast cancer by promoting its actions toward microtubule assembly and stability. Protein Cell. 2014; 5: 469-79.

18. Liu M, Yu H, Huo L, et al. Validating the mitotic kinesin Eg5 as a therapeutic target in pancreatic cancer cells and tumor xenografts using a specific inhibitor. Biochem Pharmacol. 2008; 76: 169-78.

19. Pannu V, Rida PC, Ogden A, et al. Induction of robust de novo centrosome amplification, high-grade spindle multipolarity and metaphase catastrophe: a novel chemotherapeutic approach. Cell Death Dis. 2012; 3: e346.

20. Karna P, Rida PC, Pannu V, et al. A novel microtubule-modulating noscapinoid triggers apoptosis by inducing spindle multipolarity via centrosome amplification and declustering. Cell Death Differ. 2011; 18: 632-44.

21. Sun X, Shi X, Liu M, et al. Mdp3 is a novel microtubule-binding protein that regulates microtubule assembly and stability. Cell Cycle. 2011; 10: 3929-37.

22. Tala, Sun X, Chen J, et al. Microtubule stabilization by Mdp3 is partially attributed to its modulation of HDAC6 in addition to its association with tubulin and microtubules. PLoS One. 2014; 9: e90932.

23. Yu Z, Schmaltz RM, Bozeman TC, et al. Selective tumor cell targeting by the disaccharide moiety of bleomycin. J Am Chem Soc. 2013; 135: 2883-6.

24. Kaverina I, Straube A. Regulation of cell migration by dynamic microtubules. Semin Cell Dev Biol. 2011; 22: 968-74.

25. Li D, Sun X, Zhang L, et al. Histone deacetylase 6 and cytoplasmic linker protein 170 function together to regulate the motility of pancreatic cancer cells. Protein Cell. 2014; 5: 214-23.

26. Li D, Xie S, Ren Y, et al. Microtubule-associated deacetylase HDAC6 promotes angiogenesis by regulating cell migration in an EB1-dependent manner. Protein Cell. 2011; 2: 150-60.

27. Gao J, Sun $\mathrm{L}, \mathrm{Huo} \mathrm{L}$, et al. CYLD regulates angiogenesis by mediating vascular endothelial cell migration. Blood. 2010; 115: 4130-7.

28. Goncalves J, Nolasco S, Nascimento R, et al. TBCCD1, a new centrosomal protein, is required for centrosome and Golgi apparatus positioning. EMBO Rep. 2010; 11: 194-200.

29. Shi $X$, Sun $X$, Liu M, et al. CEP70 protein interacts with gamma-tubulin to localize at the centrosome and is critical for mitotic spindle assembly. J Biol Chem. 2011; 286: 33401-8.

30. Shi $X$, Liu M, Li D, et al. Cep70 contributes to angiogenesis by modulating microtubule rearrangement and stimulating cell polarization and migration. Cell Cycle. 2012; 11: 1554-63.

31. Welcsh PL, King MC. BRCA1 and BRCA2 and the genetics of breast and ovarian cancer. Hum Mol Genet. 2001; 10: 705-13.

32. Starita LM, Machida Y, Sankaran S, et al. BRCA1-dependent ubiquitination of gamma-tubulin regulates centrosome number. Mol Cell Biol. 2004; 24: 8457-66.

33. Lafarge S, Sylvain V, Ferrara M, et al. Inhibition of BRCA1 leads to increased chemoresistance to microtubule-interfering agents, an effect that involves the JNK pathway. Oncogene. 2001; 20: 6597-606. 